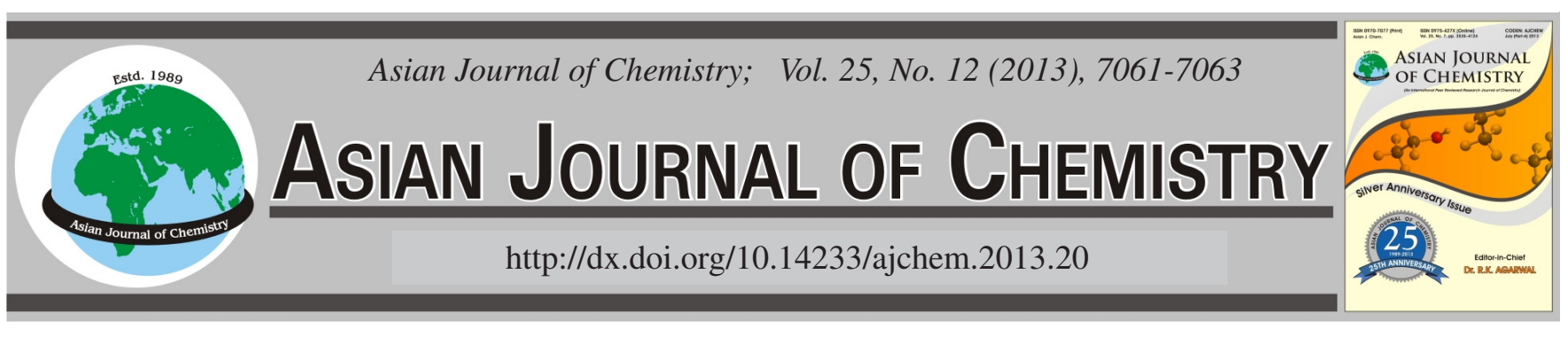

\title{
Isotopic Analysis of Uranium and Plutonium at Ultra Trace Levels Using Thermal Ionization Mass Spectrometry with Simultaneous Measurements $\dagger$
}

Jong-Ho PARK ${ }^{*}$, KAHEE JeOng and KyUSEOK SONG

Nuclear Chemistry Research Division, Korea Atomic Energy Research Institute, 989-111 Daedeok-daero, Yuseong-gu, Daejeon 305-353, Republic of Korea

*Corresponding author: Fax: +82 42 8688148; E-mail: jongho@kaeri.re.kr

AJC-13642

\begin{abstract}
A thermal ionization mass spectrometer was utilized to determine the isotopic ratios of uranium and plutonium in standard materials. Because the total amounts of uranium and plutonium for measurement were present at pg levels, the peak jumping method, which is normally used to vary the magnetic field to detect each corresponding isotope one by one, was not an appropriate method for detection. A simultaneous measurement of the isotopes of interest that adopted a multiple ion counter system was used instead to ensure high levels of analytical precision and accuracy. A correction technique using reference measurements was applied to correct mass biases and background contributions. The measurements of the target materials with the corresponding certified values, which showed that the thermal ionization mass spectrometry measurement of uranium and plutonium with the simultaneous detection method is suitable for the analysis of unknown environmental samples with high accuracy and precision.
\end{abstract}

Key Words: Uranium, Plutonium, Simultaneous measurement, Isotopic ratio, Thermal ionization mass spectrometry.

\section{INTRODUCTION}

The isotopic analysis of nuclear materials in environmental samples is greatly useful to provide crucial information about the activities that are conducted in a nuclear facility ${ }^{1}$. The analytical results may have a social impact; hence the analysis requires a high grade of accuracy and precision, which is partly ensured by utilizing thermal ionization mass spectrometry ${ }^{2}$. However, the infinitesimal quantity of the samples makes the isotope measurement very challenging. Background interference is one of the problems because the environment contains considerable amounts of uranium. In general, background interference is not considered to be a significant interference factor. However, the importance of the background contribution gradually increases up to the point where it results in a significant bias to the isotope ratio measurement as the quantity of target materials becomes smaller. The use of ultra-pure reagents and a degassing process for the thermal ionization mass spectrometry filaments prior to sample loading significantly reduced the detection of the background uranium ${ }^{3,4}$. Recently, a statistical method was developed to correct the peak tailing backgrounds for enhancing the accuracy in measuring the isotope ratios of uranium ${ }^{5}$.

Another problem arises when the amounts of samples are close to the limit of detection of the measurement techniques.
It must be noted that a micro-particle that is used for nuclear safeguards purpose usually contains only a few pg of uranium and/or a few hundred fg of plutonium. In this case, the analytical precision hardly meets the requirements with employing a normal peak jumping (or, called, SEM-jumping) method, which varies the magnetic field to detect the corresponding isotopes one by one, due to insufficient valid data points. To make the best use of the limited quantity of sample for enhanced precision, a simultaneous measurement of the isotopes of interest adopting a multiple ion counter system is required ${ }^{6}$.

In this study, isotopic ratios of uranium and plutonium in standard samples at ultra-trace levels were determined with simultaneous isotopic measurements using a thermal ionization mass spectrometry that was equipped with a multiple ion counter. Micro-particles of a standard uranium sample were also used as target samples. Reference measurements were conducted before and after the sample measurements to adjust the detector yield and to correct mass biases. The applicability of the simultaneous measurements was evaluated by comparing the measured ratios with the certified values.

\section{EXPERIMENTAL}

The experimental setup for isotopic measurements using thermal ionization mass spectrometry (TRITON, Thermo Scientific) was described in detail elsewhere ${ }^{3-5}$. A double

$†$ Presented to the 4th International Symposium on Applications of Chemical and Analytical Technologies in Nuclear Industries, Daejeon, Korea 
filament assembly consisting of two filaments was used to decouple the evaporation and the ionization of the samples.

Prior to sample loading, the filaments that were prepared from zone-refined rhenium ribbon $(0.04 \mathrm{~mm}$ and $0.7 \mathrm{~mm}$ in thickness and width, respectively, Thermo Scientific) were degassed under a vacuum $\left(\sim 10^{-7} \mathrm{mbar}\right)$ at $4.5 \mathrm{~A}$ for $60 \mathrm{~min}$. Approximately $5 \mathrm{pg}$ of a uranium standard material, U030 and $1 \mathrm{pg}$ of a standard plutonium material, REIMEP-16A, were loaded onto the filaments with a $1 \mu \mathrm{m}$ of aliquot of uranium or plutonium solution.

U030 powder for single particle loading was dispersed on a carbon planchet and then introduced in a scanning electron microscope (JSM-6610LV, JEOL) equipped with an energy dispersive X-ray spectrometer (X-Max, Oxford) for particle screening by elemental analysis. The identified micro-particles of the U030 powder with a size of approximately $2 \mu \mathrm{m}$ in diameter were then loaded on the filaments using a micromanipulation system implemented within the SEM setup.

The samples that were loaded onto the filaments were dried and fixed at $0.6 \mathrm{~A}$ and $1.8 \mathrm{~A}$, respectively. These samples were then paired with additional filaments for ionization, followed by loading into the thermal ionization mass spectrometry instrument for measurement. The continuous heating $\operatorname{method}^{7}$ was applied, where data were collected by gradually increasing the current in the evaporation filament to $4500 \mathrm{~mA}$. The multiple ion counter system consisted of 8 channeltrons, where approximately $2 \mathrm{kV}$ was applied for ion-electron conversion and the amplification of electron signals. The multiple ion counter configurations that were used for the simultaneous measurements of the uranium and the plutonium isotopes in this study are shown in Table- 1 .

\begin{tabular}{llllll}
\hline \multicolumn{5}{c}{ TABLE-1 } \\
MULTIPLE ION COUNTER CONFIGURATIONS FOR \\
SIMULTANEOUS MEASUREMENTS \\
\hline Element & IC3 & IC4 & IC5 & IC6 & IC7 \\
\hline Uranium & ${ }^{234} \mathrm{U}$ & ${ }^{235} \mathrm{U}$ & ${ }^{236} \mathrm{U}$ & & ${ }^{238} \mathrm{U}$ \\
Plutonium & ${ }^{238} \mathrm{Pu}$ & ${ }^{239} \mathrm{Pu}$ & ${ }^{240} \mathrm{Pu}$ & & ${ }^{242} \mathrm{Pu}$ \\
\hline
\end{tabular}

The detection efficiency of each channeltron was determined using the following equation to regulate the transduced ion signals by using approximately $5 \mathrm{mV}$ of rhenium ion flux prior to sample measurements.

$$
\text { Efficiency }=\frac{\text { Channeltron } \cdot \text { Intensity }(\mathrm{cps})}{\text { Faraday } \cdot \text { Intensity }(\mathrm{mV}) \times 62500(\mathrm{cps} / \mathrm{mV})}
$$

The mass bias corrections of the uranium and plutonium isotopic ratios were made based on the isotopic measurements of the reference materials. NIST U010 was used for uranium, while IRMM290A1 was used for plutonium. Mass bias correction factors $(\mathrm{C})$ were calculated as the following:

$$
\mathrm{C}=\mathrm{R}_{\text {cert }} / \mathrm{R}_{\text {meas }}
$$

where $\mathrm{R}_{\text {cert }}$ and $\mathrm{R}_{\text {meas }}$ are the certified and the measured isotope, respectively.

\section{RESULTS AND DISCUSSION}

Determination of uranium isotope ratios: Table- 2 shows the determination of the isotopic ratios of uranium in $5 \mathrm{pg}$ samples of U030 by the simultaneous measurement method (MIC) and the SEM-jumping method using thermal ionization mass spectrometry. The certified isotopic ratios of the standard materials were also shown to evaluate the accuracy of the measurements. The maximum signal intensities of ${ }^{234} \mathrm{U},{ }^{235} \mathrm{U}$, ${ }^{236} \mathrm{U}$ and ${ }^{238} \mathrm{U}$ were approximately 5, 900, 5 and 30,000 cps, respectively.

TABLE-2

ISOTOPIC MEASUREMENT RESULTS OF U030 SAMPLES USING MIC AND SEM JUMPING

\begin{tabular}{lccc}
\hline & $\begin{array}{c}\mathrm{n}\left({ }^{234} \mathrm{U}\right) / \mathrm{n}\left({ }^{238} \mathrm{U}\right) \\
\left(\times 10^{-4}\right)\end{array}$ & $\begin{array}{c}\mathrm{n}\left({ }^{235} \mathrm{U}\right) / \mathrm{n}\left({ }^{238} \mathrm{U}\right) \\
\left(\times 10^{-2}\right)\end{array}$ & $\begin{array}{c}\mathrm{n}\left({ }^{236} \mathrm{U}\right) / \mathrm{n}\left({ }^{238} \mathrm{U}\right) \\
\left(\times 10^{-4}\right)\end{array}$ \\
\hline Certified & $1.96[ \pm 0.01]$ & $3.143[ \pm 0.005]$ & $2.10[ \pm 0.01]$ \\
Meas. \#1 (MIC) & $1.92[ \pm 0.05]$ & $3.165[ \pm 0.010]$ & $2.21[ \pm 0.07]$ \\
Meas. \#2 (MIC) & $1.99[ \pm 0.04]$ & $3.140[ \pm 0.011]$ & $2.05[ \pm 0.08]$ \\
Meas. \#3 (MIC) & $1.99[ \pm 0.05]$ & $3.133[ \pm 0.011]$ & $2.08[ \pm 0.06]$ \\
Meas. \#4 (SEM) & $2.11[ \pm 0.09]$ & $3.173[ \pm 0.018]$ & $1.84[ \pm 0.10]$ \\
\hline *Numbers in parentheses indicate expanded uncertainties $\mathrm{U}=k \cdot \mathrm{u}_{\mathrm{c}}$ and \\
$k=2$
\end{tabular}

The accuracy of the measurements was less than $2 \%$ when using the simultaneous measurement (Meas. \#1 to \#3), while a greater deviation from the certified value was observed when the SEM-jumping method was used (Meas. \#4). In addition, the uncertainty, which corresponds to the measurement precision, was increased by approximately 2 times when the SEMjumping method was used. This discrepancy originates from the loss of valid data set when the SEM-jumping method was used, as only one target isotope could be detected by SEM each time, resulting in the wastage of other isotopes. For example, ${ }^{234} \mathrm{U},{ }^{235} \mathrm{U}$ and ${ }^{236} \mathrm{U}$ were annihilated when the magnetic field focused ${ }^{238} \mathrm{U}$ to the SEM (secondary electron multiplier) in jumping mode, which reduced the quantity of valid data by 4 times. The measurement accuracy and precision is not heavily influenced when the sample amount is relatively sufficient; however, the deviation is increased when small sample quantities are used, as in the case of this study.

The isotope ratios of uranium in the micro-particles were determined using the simultaneous measurement method as shown in Table- 3 . The quantity of uranium in a $2 \mu \mathrm{m}$ particle was estimated to be $30 \mathrm{pg}$ based on the assumption that the particle consisted of uranium oxides. The measurement accuracy was reasonable while the precision was enhanced in comparison with the measurement of the $5 \mathrm{pg}$ U030 samples

\begin{tabular}{|c|c|c|c|}
\hline \multicolumn{4}{|c|}{$\begin{array}{c}\text { TABLE-3 } \\
\text { ISOTOPE MEASUREMENT RESULTS OF THE U030 } \\
\text { MICRO-PARTICLES USING MIC }\end{array}$} \\
\hline & $\begin{array}{c}\mathrm{n}\left({ }^{234} \mathrm{U}\right) / \mathrm{n}\left({ }^{238} \mathrm{U}\right) \\
\left(\times 10^{-4}\right)\end{array}$ & $\begin{array}{c}\mathrm{n}\left({ }^{235} \mathrm{U}\right) / \mathrm{n}\left({ }^{238} \mathrm{U}\right) \\
\left(\times 10^{-2}\right)\end{array}$ & $\begin{array}{c}\mathrm{n}\left({ }^{236} \mathrm{U}\right) / \mathrm{n}\left({ }^{238} \mathrm{U}\right) \\
\left(\times 10^{-4}\right)\end{array}$ \\
\hline Meas. \#5 & $2.00[ \pm 0.04]$ & $3.149[ \pm 0.006]$ & $2.16[ \pm 0.04]$ \\
\hline Meas. \#6 & $1.97[ \pm 0.07]$ & $3.144[ \pm 0.009]$ & $2.19[ \pm 0.07]$ \\
\hline Meas. \#7 & $2.01[ \pm 0.03]$ & $3.133[ \pm 0.008]$ & $2.11[ \pm 0.03]$ \\
\hline Meas. \#8 & $2.02[ \pm 0.02]$ & $3.149[ \pm 0.003]$ & $2.18[ \pm 0.02]$ \\
\hline
\end{tabular}
because of the increased uranium amount.

Determination of plutonium isotope ratios: The isotope measurement results of the 1 pg REIMEP-16A samples using multiple ion counter are summarized in Table-4. The measurements of $\mathrm{n}\left({ }^{240} \mathrm{Pu}\right) / \mathrm{n}\left({ }^{239} \mathrm{Pu}\right)$ and $\mathrm{n}\left({ }^{242} \mathrm{Pu}\right) / \mathrm{n}\left({ }^{239} \mathrm{Pu}\right)$ were 
in good agreement with the corresponding certified isotopic ratios, which implies that the simultaneous measurement can estimate the isotope ratios of plutonium in an unknown sample with a high reliability, even though the uncertainty was greater than the uncertainty of the uranium measurements that were described previously.

\begin{tabular}{|c|c|c|c|}
\hline \multicolumn{4}{|c|}{$\begin{array}{c}\text { TABLE-4 } \\
\text { ISOTOPE MEASUREMENT RESULTS OF } \\
\text { REIMEP-16A SAMPLES USING MIC }\end{array}$} \\
\hline & $\begin{array}{c}\mathrm{n}\left({ }^{238} \mathrm{Pu}\right) / \mathrm{n}\left({ }^{239} \mathrm{Pu}\right) \\
\left(\times 10^{-5}\right)\end{array}$ & $\begin{array}{c}\mathrm{n}\left({ }^{240} \mathrm{Pu}\right) / \mathrm{n}\left({ }^{239} \mathrm{Pu}\right) \\
\left(\times 10^{-1}\right)\end{array}$ & $\begin{array}{c}\mathrm{n}\left({ }^{242} \mathrm{Pu}\right) / \mathrm{n}\left({ }^{239} \mathrm{Pu}\right) \\
\left(\times 10^{-4}\right)\end{array}$ \\
\hline Certified & $7.49[ \pm 0.72]$ & $6.5745[ \pm 0.0091]$ & $4.164[ \pm 0.0069]$ \\
\hline Meas. \#9 & $6.8[ \pm 0.2]$ & $6.548[ \pm 0.013]$ & $4.5[ \pm 0.4]$ \\
\hline Meas. \#10 & $6.4[ \pm 0.9]$ & $6.605[ \pm 0.046]$ & $4.5[ \pm 0.2]$ \\
\hline Meas. \#11 & $10.0[ \pm 0.4]$ & $6.565[ \pm 0.035]$ & $4.4[ \pm 0.7]$ \\
\hline
\end{tabular}

However, a considerable deviation in the measurement of $n\left({ }^{238} \mathrm{Pu}\right) / \mathrm{n}\left({ }^{239} \mathrm{Pu}\right)$ was observed. This deviation is a result of the low signal intensity of ${ }^{238} \mathrm{Pu}$, which corresponded to less than $2 \mathrm{cps}$, as well as the interference of ${ }^{238} \mathrm{U}$ background signals from the rhenium filament. It must be noted that the minimized background of ${ }^{238} \mathrm{U}$ by the filament degassing process could not be less than $1 \mathrm{cps}^{3}$, resulting in the limit of detection of ${ }^{238} \mathrm{Pu}$ to be approximately $1 \mathrm{cps}$.

\section{Conclusion}

The isotope ratios of uranium and plutonium in the U030 and the REIMEP-16A samples were determined by simultaneous measurements utilizing a thermal ionization mass spectrometry equipped with an multiple ion counter system. The measurements of the isotope ratios were in excellent agreement with the corresponding certified values, which implies that the thermal ionization mass spectrometry measurement using multiple ion counter can estimate the isotope ratios of infinitesimal contents of uranium and plutonium in unknown environmental samples with high accuracy and precision.

\section{ACKNOWLEDGEMENTS}

The authors acknowledged the financial support for this study from the Nuclear Safety and Security Commission.

\section{REFERENCES}

1. D.L. Donohue, J. Alloys Comp., 271-273, 11 (1998).

2. K.G. Heumann, S. Eisenhut, S. Gallus, E.H. Hebeda, R. Nusko, A. Vengosh and T. Wallczyk, Analyst, 120, 1291 (1995).

3. J. Park, I. Choi and K. Song, Mass Spectrom. Lett., 1, 17 (2010).

4. S. Park, J. Park, M. Lee and K. Song, Mass Spectrom. Lett., 2, 57 (2011).

5. J. Park, I. Choi, S. Park, M. Lee and K. Song, Bull. Korean Chem. Soc., 32, 4327 (2012).

6. R. Jakopic, S. Richter, H. Kuhn, L. Benedik, B. Pihlar and Y. Aregbe, Int. J. Mass Spectrom., 279, 87 (2009).

7. D. Suzuki, Y. Saito-Kokubu, S. Sakurai, C. Lee, M. Magara, K. Iguchi and T. Kimura, Int. J. Mass Spectrom., 294, 23 (2010). 